\title{
The Formability Prediction of Twinning-Induced Plasticity Steels
}

\author{
M.C. Butuc ${ }^{1, a)}$, G. Vincze ${ }^{1, \text { b) }}$ and F. Barlat ${ }^{2, c)}$ \\ ${ }^{1}$ Centre for Mechanical Technology and Automation, Department of Mechanical Engineering, University of Aveiro, \\ Campus Universitário de Santiago, 3810-193, Portugal \\ ${ }^{2}$ Graduate Institute of Ferrous Technology, Pohang University of Science and Technology, Pohang, Gyeongbuk \\ 790-784, South Korea \\ ${ }^{\text {a)} C o r r e s p o n d i n g ~ a u t h o r: ~ c b u t u c @ u a . p t ~}$ \\ b) gvincze@ua.pt \\ c) f.barlat@postech.ac.kr
}

\begin{abstract}
The proposal of this work is to predict and analyse the formability of twinning - induced plasticity steels through the Marciniak-Kuczinsky approach with emphasis on the solutions for improving the prediction results. The selected constitutive equations involve the Yld2000-2d plane stress yield function and the Swift strain-hardening power law. To understand the formability of the TWIP steel and the factors influencing it, a sensitive study on the effect of the mechanical properties of the TWIP steel on the Marciniak-Kuczinsky (MK) theory concept and the predicted forming limits is performed.
\end{abstract}

\section{INTRODUCTION}

High Mn Twinning-Induced Plasticity (TWIP) steel is a structural steel of second generation of advanced highstrength steels (AHSS) with a high strength and a superior formability attained due to the high rate of strain hardening associated with the deformation twinning phenomenon. These excellent properties of the TWIP steel with an optimum use of material can lead to important weight reduction in automotive and aerospace structures, which, in turn, can translate, into significant waste reduction and energy savings. The failure in AHSS steels has become a challenge for the steel and automotive industry. The sheet metal formability is mainly limited by the occurrence of plastic flow localization, instability and fracture. The Forming Limit Diagram method, introduced by Keeler [1] and Goodwin [2], seems to be the most advanced concept and current applied in industry to characterize the formability of sheet metals. It is essential for material selection, design and try out of the tools for deep drawing operations. Marciniak-Kuczinsky (MK) approach [3] has become one of the most important tools in predicting the sheet metal formability. The predicted limit strains strongly depend on the constitutive law incorporated in the MK analysis [47]. Concerning the formability of TWIP steels, only few researches [8-11] analysed the fracture and necking mechanics of TWIP steel. The present work aimed at analysing the ability of MK model to predict the formability of TWIP steel sheets. For this purpose, this study was devoted to the next topics: (i) simulation of the forming limits of the selected TWIP steel sheet using a software based on MK model; (ii) an analysis of the effect of the strain hardening on the prediction of the forming limits for TWIP steels; iii) searching solutions for improving the numerical results. In the MK model, proper constitutive equations, namely Yld2000-2d yield function [12] and Swift hardening law were considered. The identification of the parameters of the material models was performed for the selected TWIP steel. For validation of the model, experimental and the theoretical forming limit curves are compared. 


\section{THEORETICAL COMPUTATION OF FORMING LIMIT DIAGRAM}

The theoretical Forming Limit Diagrams presented in this work were calculated by using a modular and userfriendly code for predicting the forming limits developed by Butuc et al. [13]. The simulation of plastic instability is carried out in the framework of heterogeneous materials using the Marciniak-Kuczinsky (M-K) analysis coupled with the Theory of Plasticity. The model is based on the growth by plastic deformation within a thin sheet of an initial defect $\left(f_{0}\right)$ in the form of a groove-like, narrow band of diminished thickness. Following the Hutchinson-Neal model [14], the initial inclination angle of the band, $\psi_{0}$, with respect to the minor principal axis of the stress tensor is considered variable between 0 and $90^{\circ}$ from rolling direction. Using the method proposed by Butuc et al. [13], the computations of homogeneous and heterogeneous zones are considered independently, being their connection realized through the $\mathrm{M}-\mathrm{K}$ conditions: force equilibrium and geometrical compatibility. Through the theory of plasticity, the stress and strain state is computed. When the necking criterion is reached, the accumulated principal strains in the homogeneous zone define the forming strain limits. For each considered strain path, the limit point on the FLD is obtained through the minimization of the principal strain in the homogeneous zone, versus $\psi_{0}$. It is assumed that the material has rigid-plastic behaviour with initial yield locus shape described by the Yld2000-2d plane stress yield criterion.

The YLD2000-2d [12] plane stress yield function (acronym Yld00-2d) introduces plastic anisotropy with two linear transformations on the Cauchy stress tensor. It is expressed in terms of the deviatoric stress components as:

$\phi=\phi^{\prime}\left(\tilde{\boldsymbol{S}}^{\prime}\right)+\phi^{\prime \prime}\left(\tilde{\boldsymbol{S}}^{\prime \prime}\right)=2 \bar{\sigma}_{\mathrm{Y}}^{a}$

where $\bar{\sigma}_{\mathrm{Y}}$ is the effective stress, $a$ is an exponent connected to the crystal structure, $\phi^{\prime}$ and $\phi^{\prime \prime}$ are two isotropic functions defined by

$\phi^{\prime}\left(\tilde{\boldsymbol{S}}^{\prime}\right)=\left|\tilde{S}_{1}^{\prime}-\tilde{S}_{2}^{\prime}\right|^{a}$

$\phi^{\prime \prime}\left(\tilde{\boldsymbol{S}}^{\prime \prime}\right)=\left|2 \tilde{S}_{2}{ }_{2}+\tilde{S}_{1}{ }_{1}\right|^{a}+\left|2 \tilde{S}_{1}^{\prime \prime}+\tilde{S}_{2}{ }_{2}\right|^{a}$

$\tilde{\boldsymbol{S}}^{\prime}$ and $\tilde{\boldsymbol{S}}^{\prime \prime}$ are the linear transformations of the effective stress tensor $\boldsymbol{s}$ defined as the deviatoric part $\boldsymbol{\sigma}^{\prime}$ of the Cauchy stress:

$\tilde{\boldsymbol{S}}^{\prime}=\boldsymbol{C}^{\prime} \boldsymbol{s}, \tilde{\boldsymbol{S}}^{\prime \prime}=\boldsymbol{C}^{\prime \prime} \boldsymbol{s}$

where $\boldsymbol{C}$ ' and $\boldsymbol{C}$ ' contain the material anisotropy coefficients.

The hardening behaviour of the material is approximated by the Swift hardening law.

$\bar{\sigma}=K\left(\varepsilon_{0}+\bar{\varepsilon}\right)^{n}$

where $\bar{\sigma}_{\mathrm{Y}}$ is the yield stress, $\bar{\varepsilon}$ the effective plastic strain while $K, \varepsilon_{0}, n$ are the material constants. Each constant is calculated by fitting experimental stress/strain data.

\section{RESULTS AND DISCUSSION}

\section{Material Characterization}

The selected material is a high manganese content TWIP steel sheet sample, with the acronym TWIP980. Table 1 lists the corresponding values for the yield stresses and anisotropy factor $r$ for three uniaxial tensile directions, the balanced biaxial yield stress $\sigma_{\mathrm{b}}$ and the biaxial anisotropy coefficient $r_{\mathrm{b}}$. Table 2 lists the values of the eight anisotropy parameters for the Yld00-2d yield function calculated for different exponents, as obtained after numerical identification from the experimental data: $r_{0}, r_{45}, r_{90}, r_{\mathrm{b}}, \sigma_{0}, \sigma_{45}, \sigma_{90}, \sigma_{\mathrm{b}}$. The different exponents in the identification of the Yld000-2d coefficients are used with the purpose of analysing the effect of the sharpness of the yield locus in equibiaxial stretching in the prediction of the FLD. 
TABLE 1. TWIP 980 steel uniaxial tensile properties at $0^{\circ}, 45^{\circ}$ and $90^{\circ}$ from rolling direction

\begin{tabular}{|c|c|c|c|c|}
\hline Orientation & $\begin{array}{c}\text { Yield stress } \\
{[\mathrm{MPa}]} \\
\end{array}$ & $\begin{array}{c}\text { Biaxial yield stress } \\
\sigma_{b}[\mathrm{MPa}] \\
\end{array}$ & Anisotropy factor $r$ & $\begin{array}{c}\text { Biaxial anisotropy } \\
\text { factor } r_{b}\end{array}$ \\
\hline $0^{\circ}$ & 560 & & 0.77 & \\
\hline $45^{\circ}$ & 555 & 550 & 1.01 & 0.75 \\
\hline $90^{\circ}$ & 566 & & 1.12 & \\
\hline
\end{tabular}

TABLE 2. Coefficients of the Yld00-2d yield function for TWIP980 steel

\begin{tabular}{ccccccccc}
\hline $\boldsymbol{a}$ & $\boldsymbol{\alpha}_{1}$ & $\boldsymbol{\alpha}_{2}$ & $\boldsymbol{\alpha}_{3}$ & $\boldsymbol{\alpha}_{4}$ & $\boldsymbol{\alpha}_{5}$ & $\boldsymbol{\alpha}_{6}$ & $\boldsymbol{\alpha}_{7}$ & $\boldsymbol{\alpha}_{8}$ \\
\hline 8 & 0.965 & 1.021 & 1.018 & 0.988 & 1.025 & 1.06 & 1.012 & 0.998 \\
6 & 0.944 & 1.040 & 1.012 & 0.982 & 1.032 & 1.065 & 1.012 & 0.996 \\
5 & 0.916 & 1.066 & 1.017 & 0.976 & 1.037 & 1.062 & 1.013 & 0.994 \\
\hline
\end{tabular}

The material parameters of the Swift hardening law, identified from uniaxial tensile test at $0^{\circ}$ from $\mathrm{RD}$, are presented in table 3 . The anisotropic factor " $n$ " was determined in all strain range corresponding to the uniform plastic deformation.

TABLE 3. Material parameters of the Swift hardening law

\begin{tabular}{ccc}
\hline $\boldsymbol{K}[\mathrm{MPa}]$ & $\boldsymbol{\varepsilon}_{\mathbf{0}}$ & $\boldsymbol{n}$ \\
\hline 2250 & 0.11 & 0.65 \\
\hline
\end{tabular}

\section{Forming Limit Diagrams}

In the present study, the experimental FLDs determined by Butz et al. [10] are used. Fig. 1 shows the experimental and simulated forming limits for the TWIP980 steel under proportional loading, with the selected constitutive equations namely, the Swift hardening law and the YLD00-2d yield function, with the coefficients corresponding to " $a$ " of 6 from the table 2. It is observed a rather correct prediction of the forming limits of TWIP980 steel (the red line) but with an unusual high initial geometrical defect expressed by a very small initial defect value of 0.965 .

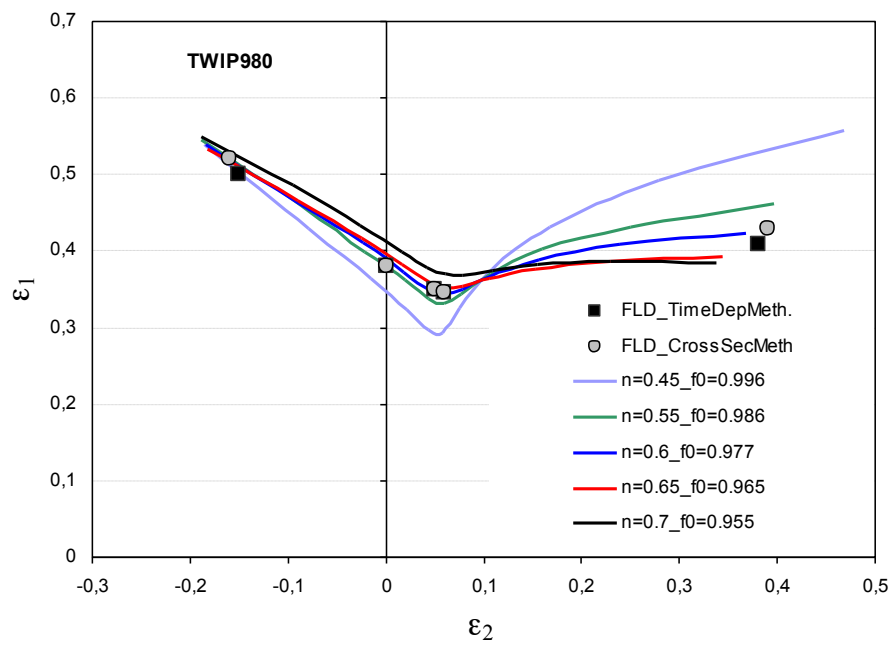

FIGURE 1. Experimental and predicted FLDs of TWIP980 steel using several values of work-hardening coefficient.

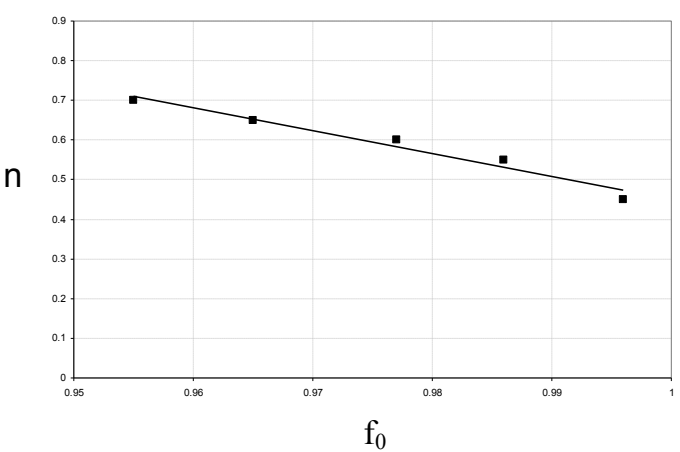

FIGURE 2. The influence of the work-hardening coefficient on the selection of the initial value of the M-K geometrical defect

In order to find the origin of such small value required in this study and since one of the most important characteristics of TWIP steel is the very high work-hardening coefficient, namely 0.65 in this case, the relation between the strain hardening coefficient and the value of the initial geometrical MK defect is analysed. For this purpose, several values between 0.45 and 0.7 were considered for the work hardening coefficient " $n$ " in the Swift 
equation to simulate the FLD for TWIP980 steel as presented in Fig. 1. To achieve the best agreement with the experimental data in uniaxial tension, the initial value of the MK geometrical defect was individually selected for each case as presented in TABLE 4. Fig. 1 shows the FLD of TWIP980 steel predicted using these values for " $n$ " and $f 0$. It is noticed that the relation between " $n$ " and $f_{0}$ is a decreasing linear function as Fig. 2 shows. According to previous studies where the value of $f_{0}$ for steel sheet were between 0.99 and 0.992 , it can be observed from Fig. 2 that " $n$ " must be smaller than 0.5 for $f_{0}$ to be greater than 0.99 . This is, in fact, applicable to most steel sheets, which usually exhibit a " $n$ " value lower than 0.3 . In conclusion, the small value of the initial MK geometrical defect is due to the very high work-hardening coefficient of the TWIP steel.

TABLE 4. The initial geometrical value for different " $n$ " for TWIP980 steel

\begin{tabular}{cccccc}
\hline $\mathbf{n}$ & 0.45 & 0.55 & 0.6 & 0.65 & 0.7 \\
$\mathbf{f}_{\mathbf{0}}$ & 0.996 & 0.986 & 0.977 & 0.965 & 0.955 \\
\hline
\end{tabular}

The experimental yield points and the predicted plane stress yield surfaces for TWIP980 steel sheet are presented in Fig. 3. The balanced biaxial yield stress used as input in the identification of the coefficients of the Yld00-2d yield function assures a great accuracy in reproducibility of yield surface on the critical region of biaxial stretching. Through the parameter "a" of the yield function, the sharpness of the yield locus in equibiaxial stretching can be controlled, fact that is very important in FLD prediction. The increase of the parameter "a" allows to an increase of the sharpness of the yield locus in equibiaxial stretching. Although for materials with face-centered cubic crystal structure FCC, like TWIP steels, is recommended $a=8$, Xu et al. [15] found that Yld00-2d with $a=6$ leads to a better prediction of the experimental data. Observing from Fig. 3 the under estimation of the forming limits in the biaxial stretching and taking into account that less sharpness of the yield locus in equibiaxial stretching is a factor promoting larger limit strains, it was selected $\mathrm{a}=5$ in the simulation, in order to reduce the sharpness of the yield surface. Fig. 4 shows the Forming limit Diagrams of TWIP980 steel simulated with different values of "a" namely 5 , 6 and 8. A significant improvement of the accuracy of the predicted FLD of TWIP980 steel was achieved using a=5. If the $f_{0}$ value is slightly modified to 0.963 the predicted FLD reproduces almost perfectly the experimental data. These results could be attributed to the twinning that appeared in the deformation of the TWIP steels, which might influences, the sharpness of the yield locus [15] by decreasing its curvature.

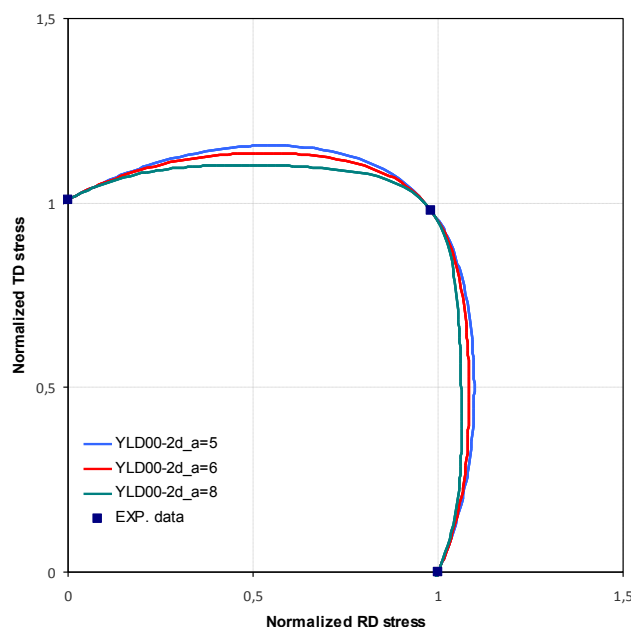

FIGURE 3. Experimental and predicted Yield Surface of Twip980 Steel.

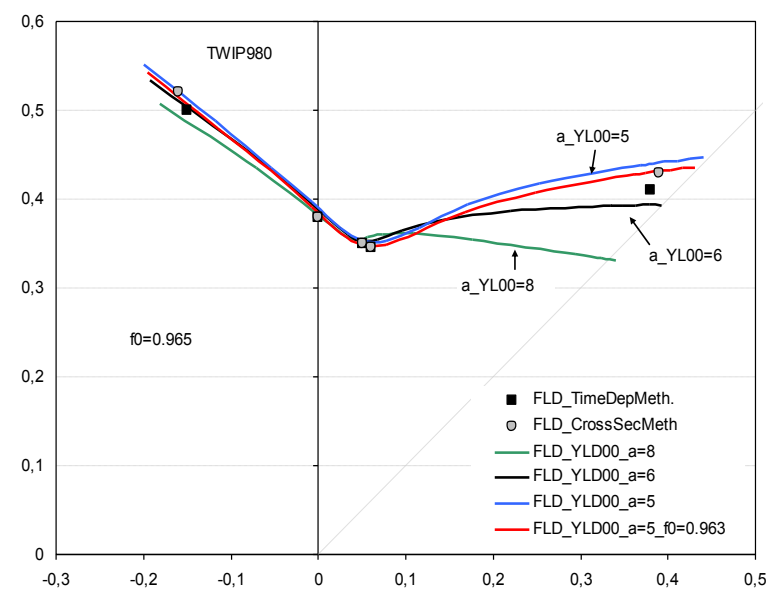

FIGURE 4. The influence of sharpness of the yield surface on FLDs of TWIP980 steel predicted with YLD2000$2 \mathrm{~d}$ using different values of parameter "a"

\section{CONCLUSIONS}

MK theory has been used to predict the forming limits for TWIP steel sheets under linear strain paths. A good agreement between experimental and theoretical results has been found. The accuracy on the prediction of the 
forming limits is due to the ability of the selected constitutive equations, namely Yld2000-2d yield function and Swift strain-hardening power law, to describe the TWIP steel behaviour. The present analysis shows the great importance of the ability of the yield function to reproduce the sharpness of the yield surface in the biaxial stretching region for the correctness of the prediction of the FLDs, emphasizing also the key role of the correct identification of the parameters of the material. MK theory seems to be able to predict the forming limits of TWIP steel with the particularity of using a very high initial geometrical defect due to the very high work-hardening coefficient of the TWIP steel. Anyway, finding the mechanisms of TWIP steels that lead to fracture without much of a diffuse necking should be a priority for future studies.

\section{ACKNOWLEDGMENTS}

The authors acknowledge support from the Operational Program for Competitiveness and Internationalization, in its FEDER/FNR component and the Portuguese Foundation of Science and Technology (FCT), in its State Budget component (OE) through projects POCI-01-0145-FEDER-032466, UID/EMS/00481/2013 and CENTRO-01-0145FEDER-022083.

\section{REFERENCES}

1. S.P. Keeler, Society of Automotive Engineers, Technical paper No. 650535 (1965).

2. G.M. Goodwin, Society of Automotive Engineers, Technical paper no. 680093 (1968).

3. Z. Marciniak and K. Kuczynski, Int. J. Mech. Sci. 9, 609-620 (1967).

4. M.C. Butuc, A. Barata da Rocha, J.J. Gracio, J. Mat. Proc. Tech., 142, Issue 3, 714-724 (2003).

5. D. Banabic, D.S. Comsa, P. Jurco, S. Wagner, P. Van Houtte, 2004. Proc. of the $7^{\text {th }}$ Esaform Conference on Material Forming, 455-458, (2004).

6. H. Aretz, Int J. Plast. 23, 798-840, (2007).

7. J. Cao, H. Yao, A. Karafillis and M.C. Boyce, Int J of Plast. 16, 1105-1129 (2000).

8. K. Chung, K. Ahn, D.-H. Yoo, K.-H. Chung, M.-H. Seo, S.-H. Park, Int J of Plast. 27, 52-81 (2011).

9. Y. Lou, H. Huh, S. Lim and K. Pack, Int. J. of Solids and Structures, 49, 3605-3615 (2012).

10. A. Butz, M. Zapara, A. Erhart, D. Croizet, N. Stenberg, J. Hagstrom, D. Helm, M. Schneider, M. Kampczyk, A. Haufe, M. Biasutti, IDDRG 2014 Conference Proceedings, (2014).

11. N. Habibi, A. Zarei-Hanzaki, H.-R. Abedi, J. Mat. Proc. Tech., 224, 102-116 (2015).

12. F. Barlat, J. C. Brem, J. W. Yoon, K. Chung, R. E. Dick, D. J. Lege, F. Pourboghrat, S.-H. Choi, E. Chu, Int. J. Plast. 19, 1297-1319 (2003).

13. M.C. Butuc, C. Teodosiu, F. Barlat, J.J. Grácio, European J. of Mech. /A Solids, 30, 532-546 (2011).

14. R.W. Hutchinson and K.W. Neal, in Mechanics of Sheet Metal Forming, 269-285 (1978).

15. L. Xu, F. Barlat, M.-G. Lee, Scripta Materialia, 66, Issue 12, 1012-1017 (2012). 